\title{
Síndrome de Brown-Séquard secundario a traumatismo craneoencefálico leve en paciente con canal cervical estrecho asintomático. Reporte de un caso y revisión de la literatura médica
}

\author{
Bernardo Urrutia-Stamatio ${ }^{1}$, Juan Carlos Betancourt-Villaruel Aldana¹, Maribel López-Fernández¹, \\ Karen Ivette Ocampo-Bustos ${ }^{1}$ y Joel Padilla-Rubio ${ }^{2}$ \\ ${ }^{1}$ Servicio de Medicina Interna, Hospital General de Zona Norte de Puebla "Bicentenario de la Independencia», Puebla; ${ }^{2}$ Servicio de Neurología, \\ Hospital Aranda de la Parra, León, Gto.. México
}

\begin{abstract}
Resumen
Introducción: El síndrome de Brown-Séquard es una entidad patológica poco común, reportada tan sólo como un 1-4\% de todos los síndromes medulares. Se han descrito varias etiologías como traumatismo, siringomelia, hernia de disco, neoplasia de la médula espinal,enfermedad desmielinizante, mielitis infecciosa e isquemia de la médula espinal.Reporte de caso: Mujer de 58 años que acude a Urgencias por caída desde su propia altura sin pérdida del estado de alerta, pero con hemiplejia izquierda, por lo que se realiza tomografía de cráneo simple a las 3 y $48 \mathrm{~h}$ del inicio de la sintomatología, sin reportar evidencia de imágenes hipodensas o hiperdensas. En el Servicio de Urgencias se le diagnostica enfermedad cerebrovascular de tipo isquémica, por lo que es ingresada en la planta de Medicina Interna para continuar con el protocolo de estudio y tratamiento. En la exploración física se encuentra hemiplejia izquierda, ausencia de propiocepción de hemicuerpo izquierdo y termoalgesia abolida en hemicuerpo derecho, integrando todo ello un síndrome de Brown-Séquard. Se solicita tomografía computarizada simple de columna cervical y resonancia magnética con gadolinio, que reportan fusión de cuerpos vertebrales en C4-C5 y C7-T1, con espondilolistesis de C5 y canal cervical estrecho. Conclusión: El síndrome de Brown-Séquard es una entidad poco reportada, siendo aún más rara su asociación a la fusión de cervicales con canal cervical estrecho. Para su correcto diagnóstico resulta fundamental realizar una exploración física neurológica completa.
\end{abstract}

Palabras clave: Brown-Séquard. Canal cervical estrecho. Traumatismo craneoencefálico leve.

\section{Correspondencia:}

Bernardo Urrutia-Stamatio

Servicio de Medicina Interna

Hospital General de Zona Norte de Puebla

"Bicentenario de la Independencia»

88 poniente, Sin número

Unidad habitacional Infonavit San Pedro

C.P. 72200 , Puebla, Pue., México

Fecha de recepción: 07/02/18

Fecha de aceptación: 15/08/2018

DOI: 10.24875/RMN.M18000009
Disponible en internet: 13-11-2018 Rev Mex Neuroci. 2018;19(5):64-67 www.revmexneuroci.com 1665-5044/@ 2018. Academia Mexicana de Neurología A.C. Publicado por Permanyer México. Este es un artículo Open Access bajo la licencia CC BY-NC-ND (http://creativecommons.org/licenses/by-nc-nd/4.0/). 


\title{
Brown-Séquard Syndrome Secondary to Mild Traumatic Brain Injury in Patient with Asymptomatic Cervical Spinal Stenosis: Case Report and Literature Review
}

\begin{abstract}
Introduction: The Brown-Sequard syndrome is a rare pathological entity, reported as 1-4\% of all spinal syndromes. Several etiologies have been described, such as trauma, syringomyelia, disc herniation, spinal cord neoplasia, demyelinating disease, infectious myelitis and spinal cord ischemia. Case Report: A 58-year-old female patient who came to the emergency room due to falling from her own height without loss of alertness but with left hemiplegia. A simple computed tomography scan of the brain was performed at 3-48 $h$ after without evidence of hypo or hyperdense images. Diagnosed in the emergency department as ischemic stroke, it entered into internal medicine floor to continue study and treatment protocol; Physical examination revealed left hemiplegia, absence of left hemisphere proprioception and abolished thermoalgesia in the right side of the body, integrating a Brown-Sequard syndrome. Simple computed tomography of the cervical spine and magnetic resonance with gadolinium are requested, which report fusion of vertebral bodies in C4-C5 and C7-T1 with C5 spondylolisthesis and narrow cervical canal. Conclusion: Brown Sequard syndrome is a disease with few cases reported, being even more rare its association to the fusion of cervical vertebrae with stenosis cervical canal; important for its timely diagnosis a complete neurological physical examination.
\end{abstract}

Key words: Brown-Sequard. Stenosis cervical canal. Mild traumatic brain injury.

\section{Introducción}

En 1846, C.E. Brown-Séquard describió por primera vez este síndrome mientras realizaba experimentos en animales en los que investigaba las vías de sensibilidad en la médula espinal'. En 1849 se identifica por primera vez clínicamente a un paciente con hemisección traumática secundaria a herida por arma blanca en la médula espinal ${ }^{2}$.

El síndrome de Brown-Séquard, también conocido como síndrome de hemisección medular, es producto de una interrupción de las vías ascendentes y descendentes.

Se caracteriza por pérdida de la función motora ipsilateral secundaria a interrupción del tracto corticoespinal, pérdida de la sensibilidad profunda y de la propiocepción debida a afección de los cordones posteriores, y pérdida contralateral de la sensibilidad termoalgésica por disfunción del tracto espinotalámico ${ }^{3}$.

La espondilólisis cervical se presenta hasta en un $50 \%$ en personas mayores de 40 años y puede causar frecuentemente estenosis del canal cervical, por lo que este tipo de pacientes tiene mayor riesgo de presentar lesiones de la médula espinal, ya que ésta no se puede mover libremente en el canal espinal, por lo que un traumatismo leve cervical puede causar lesiones devastadoras 4 .

\section{Reporte de caso}

Mujer de 58 años, originaria de Puebla y de profesión campesina. Antecedentes de relevancia: catarata bilateral de dos años de diagnóstico.
El 7 de enero de 2018 sufre una caída desde su propia altura sin pérdida del estado de alerta, aunque inicia hemiplejia izquierda, por lo que es trasladada al Servicio de Urgencias a las $3 \mathrm{~h}$ posteriores a su caída. A su llegada al servicio se realizan dos tomografías computarizadas de cráneo simple -a su ingreso y a las $48 \mathrm{~h}$ posteriores-, que no muestran evidencias de hiperdensidad o hipodensidad. Es ingresada en la planta de Medicina Interna, donde en la exploración física se encuentra: paciente mujer de edad aparente a la real; funciones mentales: despierta, fija, dirige y mantiene la atención, orientada en las cuatro esferas, no presenta disartria ni alteraciones en el lenguaje, y el resto de aspectos sin anormalidades; nervios del cráneo: I, sin alteraciones; II, campimetría y agudeza visual no valorables, y catarata bilateral; III, IV y VI, no valorables; V, reflejo corneal bilaterales, y sensibilidad y fuerza conservadas; VII, sin alteraciones; VIII, Rinné y Weber sin alteraciones; IX y X, con úvula central reflejo nauseoso bilateral; $\mathrm{XI}$, tono y trofismo de esternocleidomastoideo y trapecio conservado, fuerza conservada; XII, lengua central con movimiento conservado. Respecto a las características motoras, se observa: hemiplejia izquierda, fuerza 5/5 en hemicuerpo derecho; reflejos de estiramiento muscular +++/++++ en hemicuerpo izquierdo $\mathrm{y}++/++++$ en hemicuerpo derecho; respuesta plantar extensora izquierda; respuesta plantar flexora derecha; Hoffman y Trommer presentes en hemicuerpo izquierdo. Sobre el tono-trofismo, se detecta espasticidad en hemicuerpo izquierdo. Otros datos que se observan son: sensibilidad, pérdida de termoalgesia en 


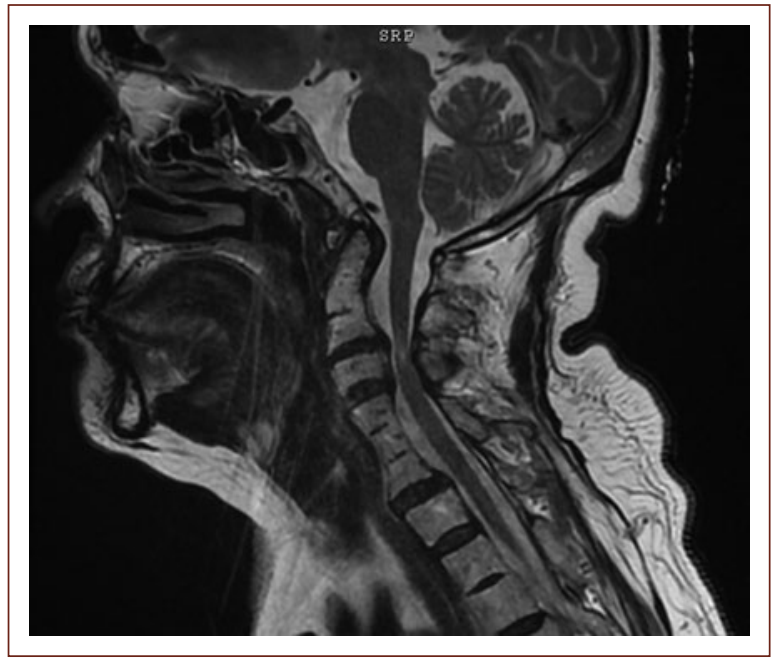

Figura 1. Imagen de resonancia magnética corte sagital secuencia T2 donde se observa fusión de cuerpos vertebrales C4-5 y C7-T1 con espondilolistesis de C5 y canal cervical estrecho con presencia de hiperintensidad.

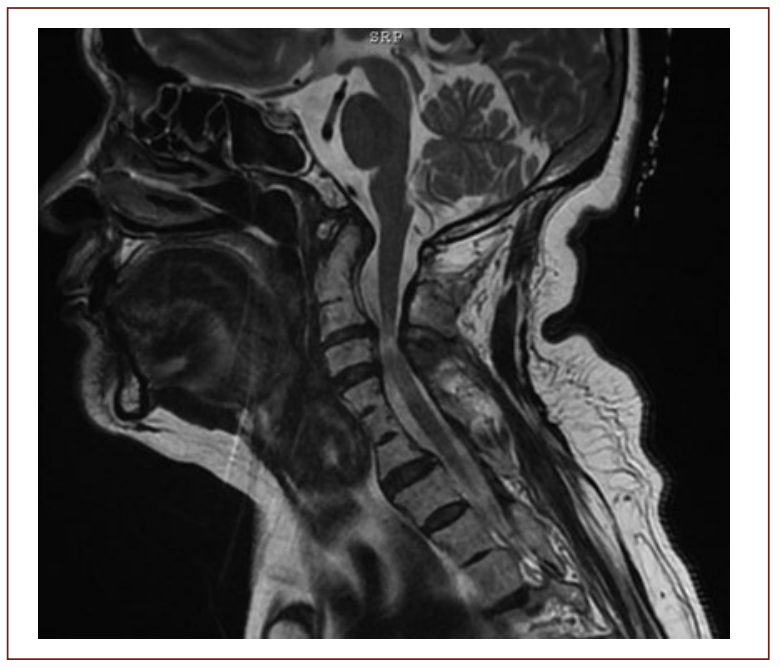

Figura 2. Imagen de resonancia magnética corte sagital secuencia T2 donde se observa fusión de cuerpos vertebrales C4-5 y C7-T1 con espondilolistesis de C5 y canal cervical estrecho con presencia de hiperintensidad. hemicuerpo derecho desde $\mathrm{C} 6$, cerebelo no valorable, movimientos anormales ausentes, marcha no valorable, atávicos ausentes, signos meníngeos ausentes y sistema nervioso autónomo sin alteraciones.

Se inició tratamiento con metilprednisolona y se solicita resonancia magnética simple y contrastada de columna cervical, en las cuales se observa fusión de cuerpos vertebrales en C4-C5 y C7-T1, con espondilolistesis de $\mathrm{C} 5$ y canal cervical estrecho.

La paciente es enviada al Hospital de Traumatología y Ortopedia Doctor y General Rafael Moreno Valle para ser valorada por el Servicio de Cirugía de Columna, donde se le diagnostica síndrome de Brown-Séquard secundario a canal medular estrecho y se le ofrece tratamiento quirúrgico; sin embargo, al no contar con recursos económicos, la paciente egresa por máximo beneficio (Figuras 1-3).

\section{Discusión}

Se decide realizar reporte de este caso clínico porque, según la bibliografía médica consultada, sólo existe un caso documentado en la literatura médica mexicana del síndrome de Brown-Séquard, el cual fue secundario a una hernia cervical de $\mathrm{C} 5-\mathrm{C} 6^{5}$. Tomando como ejemplo nuestro caso, se podría pensar que la baja incidencia de esta enfermedad es secundaria a la baja sospecha de la misma y la pobre experiencia en la realización de una exploración física neurológica completa por el personal de salud fuera del área de neurología.

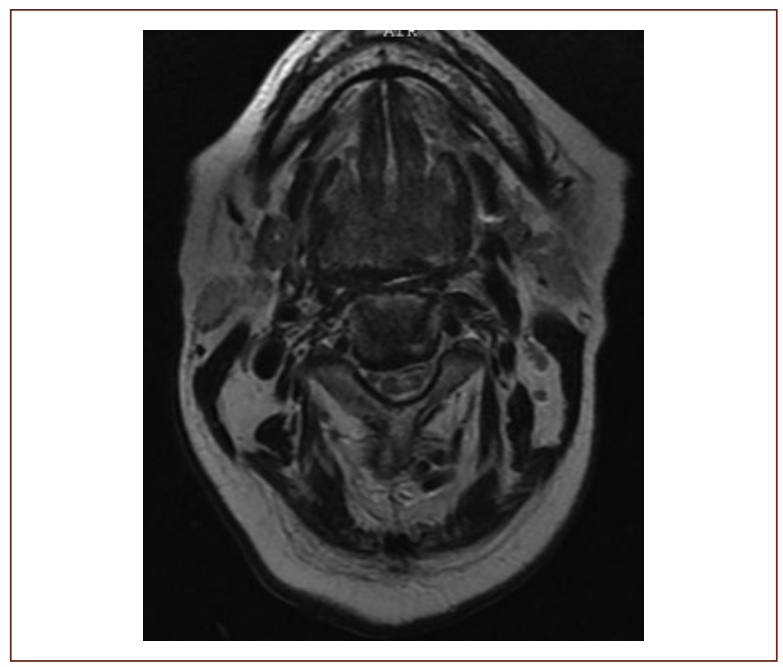

Figura 3. Imagen de resonancia magnética corte axial secuencia T2 a nivel cervical C4 con presencia de hiperintensidad en la medula espinal.

En un estudio retrospectivo realizado en Nantes (Francia), con 63 pacientes hospitalizados por lesión traumática cervical aguda y canal cervical estrecho desde enero de 2000 hasta diciembre de 2012, se identificó que el nivel neurológico de la lesión más frecuente fue en C4 (35.7\%) y C5 (28.6\%). Se observaron síndromes clínicos en un $78.6 \%$, siendo el más frecuente el síndrome de Brown-Séquard, con un 30.9\% ${ }^{4}$.

Para la realización del diagnóstico algunos autores han reportado que el protocolo de examen clínico, 
tomografía computarizada e imagen de resonancia magnética en el traumatismo de la médula espinal tiene una sensibilidad del $99 \%$ y una especificidad del $100 \%{ }^{6}$. Miranda, et al. han realizado un estudio retrospectivo en pacientes con síndrome de Brown-Séquard buscando correlacionar los hallazgos radiológicos con la presentación clínica y el pronóstico. Los autores encontraron que la presencia de hiperintensidades en la secuencia T2 correspondía al nivel neurológico de la lesión, aunque no mostraba concordancia con el grado de deterioro neurológico ${ }^{7}$.

En cuanto al tratamiento médico, es controversial el uso de esteroides intravenosos. En las últimas guías de manejo de una lesión espinal aguda del Congreso de Neurocirujanos del año 2013, el uso de metilprednisolona no cuenta con nivel de evidencia ni grado de recomendación; sin embargo, existen múltiples publicaciones en las que los pacientes han presentado mejoría clíni$\mathrm{ca}^{8,9}$. Tal es el caso de nuestra paciente, quien mostró mejoría de la sensibilidad tras la administración de metilprednisolona; sin embargo, el déficit motor persistió.

Dadas las limitaciones de nuestro hospital, la paciente fue referida al Hospital de Traumatología y Ortopedia Doctor y General Rafael Moreno Valle, donde fue valorada por el Servicio de Cirugía de Columna, que ofrece a la paciente tratamiento quirúrgico, el cual no fue aceptado por falta de recursos económicos, por lo que la paciente egresó por máximo beneficio.

\section{Conclusión}

El síndrome de Brown-Séquard es una entidad poco reportada en la bibliografía médica mundial, siendo aún más rara su asociación a la fusión de cervicales en un paciente con canal cervical estrecho. Puede generar un gran grado de discapacidad a los pacientes, por lo cual es importante un diagnóstico correcto, siendo parte fundamental del mismo una exploración física neurológica completa. Hacen falta más estudios que apoyen el uso de esteroides en este tipo de enfermedades para poder otorgarles cierto grado de recomendación.

\section{Declaración de conflictos de interés}

Los autores declaran no presentar conflictos de interés.

\section{Fuente de financiamiento}

Este reporte de caso no tuvo ningún financiamiento.

\section{Bibliografía}

1. Roth EJ, Park T, Yarkony GM, Pang T., Lee M. Y., Traumatic cervical Brown-Sequard and Brown-Sequard-plus syndromes: the spectrum of presentations and outcomes. Paraplegia 29 (9), 1991, 582-9.

2. Tattersall R., Turner B. Brown-Séquard and his syndrome, Lancet 356 , 2000, 61-63.

3. Miranda, P., Gomez, P., Alday, R. Kaen A., Ramos A., Brown-Sequard syndrome after blunt cervical spine trauma: clinical and radiological correlations, Eur Spine J 16 (8) 2007, 1165-1170

4. Y. Ronzi, B. Perrouin-Verbe, O. Hamel et al, Spinal cord injury associated with cervical spinal canal stenosis: Outcomes and prognostic factors, Ann Phys Rehabil Med 1134, 2017, 1-6

5. Padilla-Vazquez F., Escobar-de la Garma V. H., González-Fajardo M.B. Sindrome de Brown Sequard en una hernia discal cervical, Archivos Neurociencias 18 (2), 2013, 104-107.

6. Ryken T.C., Hadley M.N., Walters B.C. et al Radiographic assessment, Neurosurgery 72, 2013, 54-72,

7. E. Lim et al. Traumatic atypical Brown-Sequard syndrome: case report and literature review Clinical Neurology and Neurosurgery 105, 2003, 143-145.

8. Hurlbert R.J., Hadley M.N., Walters B.C. et al. Pharmacological Therapy for Acute Spinal Cord Injury, Neurosurgery 72, 2013, 93-105,

9. MH Pouw et al, Traumatic cervical Brown-Sequard-plus síndrome, Spinal Cord 48, 2010, 614-618 